\title{
Minimal Surface Form Finding and Visualization using Stretched Grid Method
}

\author{
E.V. Popov ${ }^{1, A}$, T.P. Popova ${ }^{2, B}$ \\ A Nizhegorodsky State Architectural and Civil Engineering University \\ B National Research University Higher School of Economics \\ ${ }^{1}$ ORCID: 0000-0002-3058-2369, popov_eugene@list.ru \\ 2 ORCID: 0000-0002-1351-222X, tatpop@list.ru
}

\begin{abstract}
Geometric and computational approach based on the theory of Stretched Grid Method (SGM) has been developed for the minimal surfaces form finding. For example, SGM is advantageous for engineering problem solution relative to an arbitrary tensile fabric structures design in comparison with other approaches. The SGM fundamental properties concerning convergence of the method and applicability have been proved in this paper. The proof is based on the theorem about the first surface quadratic form behavior together with convergence to minimum surface area. An efficient computer procedure based on the approach described in this paper has been worked out. The procedure is included into CAD system aimed at tensile fabric structures designing.
\end{abstract}

Keywords: Minimal surface, first surface quadratic form, Stretched Grid Method, Tensile fabric structure.

\section{Introduction}

The theory of minimal surfaces in three-dimensional Euclidean space has its roots in the calculus of variations developed by Euler and Lagrange in the XVIII century and in later investigations in XIX century by Enneper, Scherk, Schwarz, Riemann and Weierstrass. Since then, many great mathematicians have contributed a lot to this theory. Most of the activity in minimal surface theory in those days was focused almost exclusively on Plateau's problem [12]. However, in most cases the Plateau's problem of analytic solution is impossible because of the lack of a surface analytic representation in the majority of cases.

At the same time, the theory of minimal surfaces form finding is relevant for some architectural engineering problems solution. Since the middle of XX century, advanced types of structures have constituted an important research field in architecture and engineering. Amongst them are tensile fabric structures. They are light, elegant and effective structures, whose applications range from large stadium roofs and high-rise building walls to pneumatic furniture or aerospace equipment. Tensile fabric structures are characterized by profusion of possible equilibrated initial configurations, and, for this reason, it is difficult to define their geometric shape a priori. The design of a tensile fabric structure involves the determination of an initial equilibrated configuration or viable configuration, which encompasses the structure's shape and the associated stress field. The viable configuration must accommodate both architectonic (form and function) and structural requirements (strength and stability). The design of tensile fabric structures is consequently integrated in their engineering analysis, in a process that includes procedures for shape finding, patterning and load analysis. The most common way to form finding of doubly curved fabric structures is to minimize their area because they naturally form a minimal surface-the surface with minimal area like a soap film. It is well known that a surface like a soap film has a uniform stress in every direction and low material consumption at the same time that are very attractive 
properties for fabric structures. Therefore, the minimal surfaces form finding is a key concept in the design of tensile fabric structures in the majority of cases.

\section{Problem Formulation}

In practice, typical tensile structure surface is represented by a facet shell model in the form of a triangle mesh as it is shown in Fig. 1. In practice, the typical surface of the stretching structure is represented by a faceted shell model.

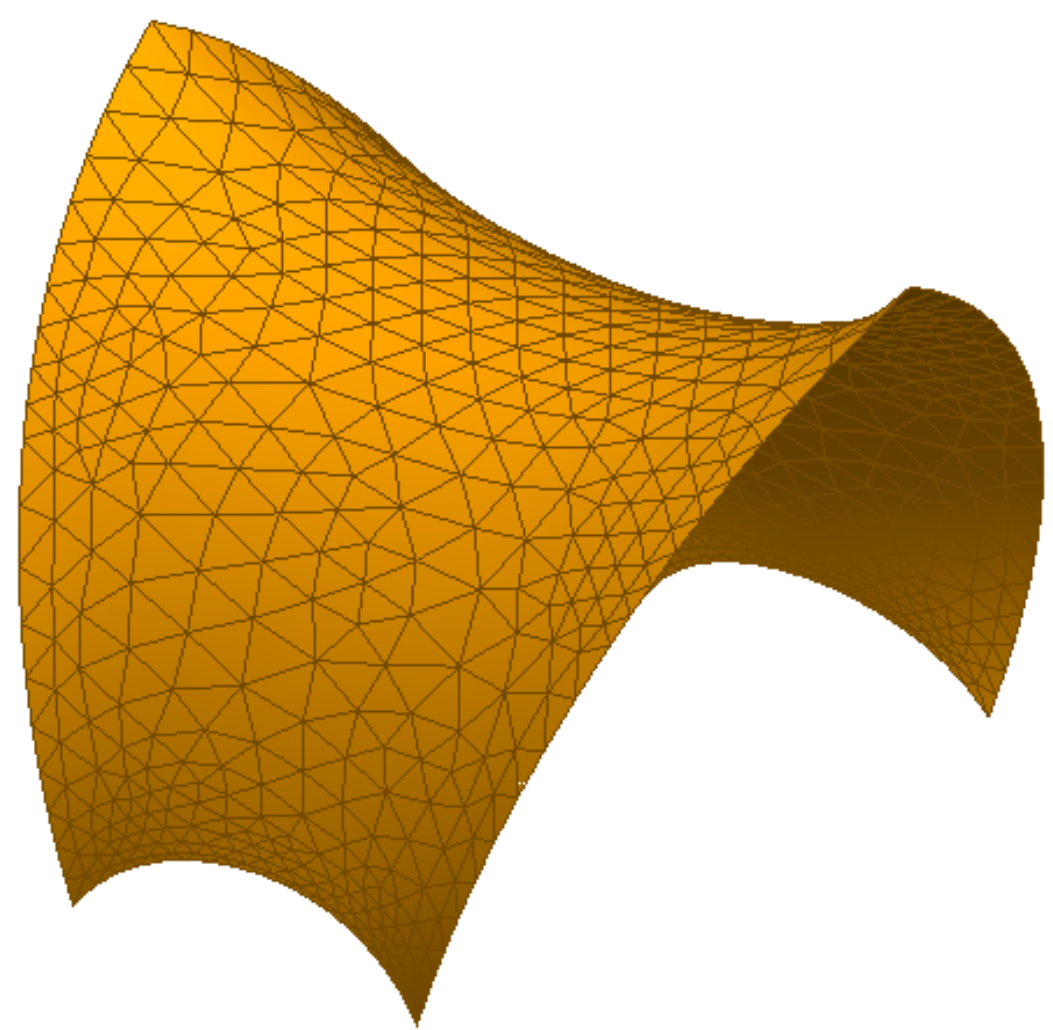

Figure 1. Typical surface discrete model

Nowadays the solution to the minimum surface problem is based on discrete numerical methods such as FEM and others. The constitutive problem of form finding for fabric tension structures on minimal surface basis is formulated in [3 - 6]. Some computer methods for computing the initial equilibrium shapes of tension structures are presented in these works. All methods use different large-displacement finite element formulations. The alternative approximated approach to the problem solution is based on the total energy balance of a nodal system as it is described in [7]. Due to its physical meaning this approach can be called the Stretched Grid Method (SGM). Usually, meteorologists use SGM for weather forecasting [8]. We use SGM to design tents and other tensile structures. The main idea of SGM is to obtain the total energy balance of grid-nodal structure. It allows substituting the complex solution of the non-linear FEM problem by very efficient linear SGM formulation.

However, it seems necessary to consider the problem in more detail because there are some dubious points concerning the approach convergence and the mathematics proof. The approach described in [7] is based on the following function minimum finding

$$
\Pi=D \sum_{j=1}^{n} R_{j}^{2},
$$

where $n$ - total number of segments in the network,

$R j$ - the length of segment number $j$,

$D-$ an arbitrary constant (does not influence the final result). 
As it is shown in [7] the applicability of eqn. (1) to minimal surface problem solution could be proved by the Heronian transformation for a single triangle in $3 \mathrm{D}$ space providing

$$
L=\frac{1}{4} \sum_{1}^{3} a_{i}^{2}>S_{\Delta}=\frac{1}{4} \sqrt{\left[\left(\sum_{1}^{3} a_{i}^{2}\right)^{2}-2 \sum_{1}^{3} a_{i}^{4}\right]},
$$

where $S_{\Delta}$ - the area of one triangle,

$a_{i}$ - the length of triangle edge number $i$

at any variable nodal vector $\Delta x_{j}$. One can affirm that if vector $\Delta x_{j}$ supplying the minimum to the function $L$ is found, it will also give the minimum (or only stable state) to the function $S_{\Delta}$. If we write further the total $3 \mathrm{D}$ surface area with a triangular grid as a sum of areas of all triangles

$$
S_{\Sigma}=C \sum_{k=1}^{n} S_{\Delta_{k}}
$$

we may also notice here that the following expression is always true due to (2), i.e.

$$
\Pi>S_{\Sigma} .
$$

Thus, as it was shown in [7] the minimum surface problem is focused on function (3) minimum finding and may be replaced by function (1) minimum problem solution.

However, the sample presented in Fig.2 illustrates some discrepancies concerning the above proof. Let's specify this problem. It is a non-planar rectangle simulated by four triangles.

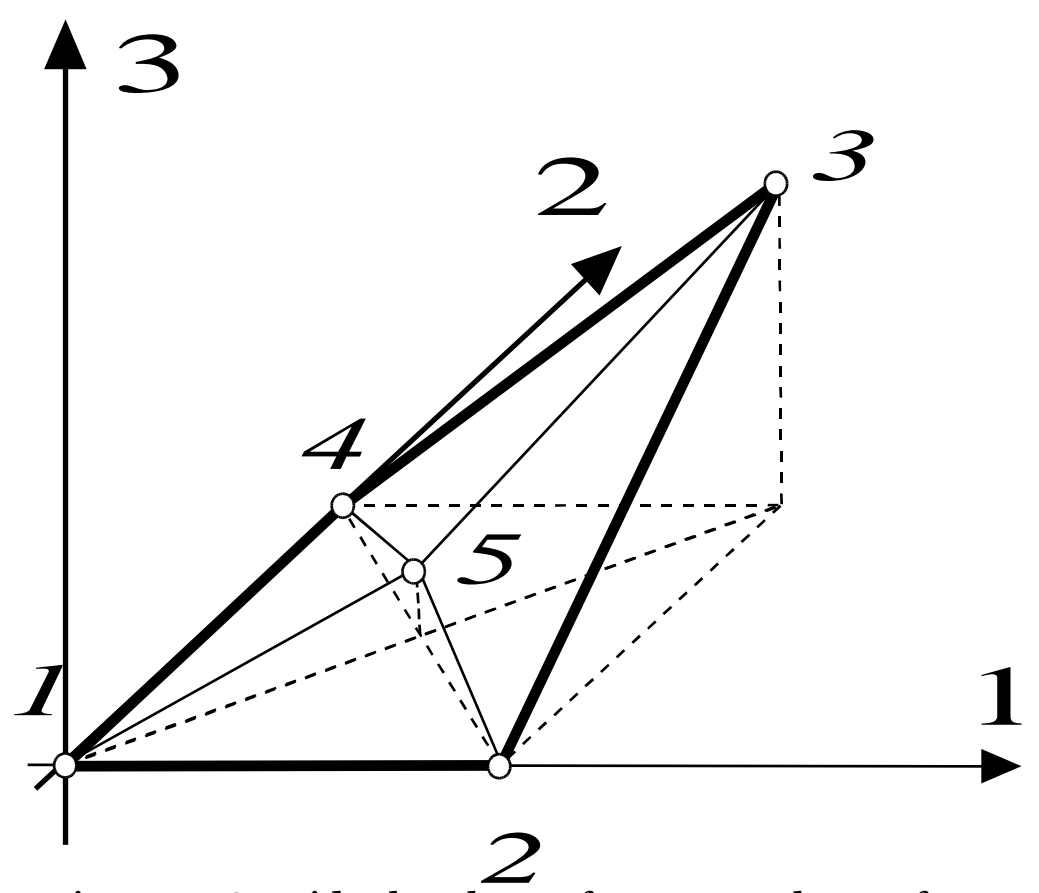

Figure 2. Outside the plane of a rectangular surface

The boundary nodal co-ordinates are presented in table below The nodal co-ordinates

\begin{tabular}{|l|l|l|l|}
\hline Node & $\mathrm{X}_{1}$ & $\mathrm{X}_{2}$ & $\mathrm{X}_{3}$ \\
\hline 1 & $\mathrm{O}$ & $\mathrm{O}$ & $\mathrm{O}$ \\
\hline 2 & 1 & $\mathrm{O}$ & $\mathrm{O}$ \\
\hline 3 & 1 & 1 & 1 \\
\hline 4 & $\mathrm{O}$ & 1 & $\mathrm{O}$ \\
\hline
\end{tabular}

When minimizing eqn. (1) one can find the following co-ordinates of node $5: \mathrm{X}_{1}=0.5, \mathrm{X}_{2}=0.5$, $\mathrm{X}_{3}=0.25$. Taking into account these co-ordinates, we may calculate a total rectangular area as 1.309017. On the other hand, using eqn. (3) we may calculate $X_{1}=0.500262, X_{2}=0.500262$, 
$\mathrm{X}_{3}=0.207376$ with a total rectangular area 1.306563. It means functions (1) and (3) have their minima at different vectors $\Delta x_{j}$ and the conclusion made in [7] is either wrong or needs an additional proof aimed at finding any limitations of the described approach.

\section{Surface Area Calculation}

First of all, we need to prove that the area of the facet surface model converges to the area of a smooth surface due to the grid condensing. Let's assume there is an arbitrary closed nonplane contour bounding an arbitrary 3D surface. Assume further that the surface is approximated by a number of plane triangles so that their nodes are situated precisely on the surface. Let's study a single triangle of such grid in more detail (see Fig.3).

The reasoning described in this Section is a trial to evaluate the difference between areas of real and plane triangles. Let us assume that the difference can be expressed in the following way

$$
\tilde{S}-\Delta=\gamma
$$

where $\tilde{S}$ - the area of real curved surface triangle,

$\Delta$ - the area of plane triangle on the same nodal basis,

$\gamma$ - the difference between areas.

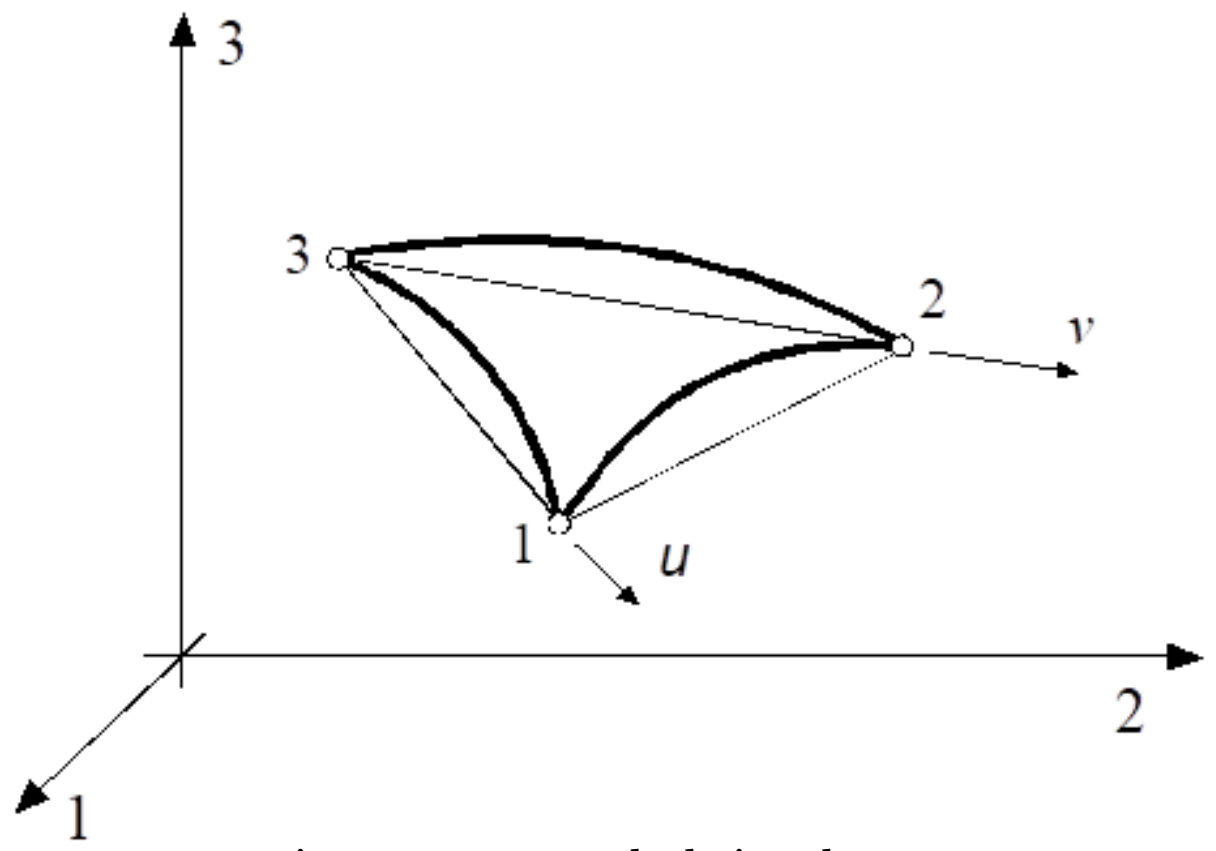

Figure 3. To area calculation element

As shown in Fig 3 the local co-ordinate system $u, v$ associated with the surface of plane triangle allows to write the following linear equations for triangle co-ordinates

$$
x_{i}=x_{i 3}+\left(x_{i 1}-x_{i 3}\right) \cdot u+\left(x_{i 2}-x_{i 3}\right) \cdot v
$$

where $x_{i j}$ - nodal co-ordinates of the plane triangle on axis $i$,

$i=1,2,3$ - number of axes,

$j=1,2,3$ - number of mangle node.

However, using the form for finite increments we may write the equation of linear mapping for triangle points in the following way

$$
\hat{x}_{i}=x_{i 3}+\frac{\partial x_{i}\left(u^{0}\right)}{\partial u} \Delta u+\frac{\partial x_{i}\left(v^{0}\right)}{\partial v} \Delta v
$$

where $u^{0}$ and $v^{0}$ - mean derivatives at node number 3 (see Fig. 3).

A non-linear mapping of a similar point within a curved triangle may be presented as 


$$
\tilde{x}_{i}=x_{i 3}+\frac{\partial x_{i}(u)}{\partial u} \Delta u+\frac{\partial x_{i}(v)}{\partial v} \Delta v+\alpha_{i}
$$

where $\alpha_{i}$ - non-linear residual of the mapping which is equal to

$$
\alpha_{i}=\left(\frac{\partial x_{i}\left(u^{*}\right)}{\partial u}-\frac{\partial x_{i}\left(u^{0}\right)}{\partial u}\right) \Delta u+\left(\frac{\partial x_{i}\left(v^{*}\right)}{\partial v}-\frac{\partial x_{i}\left(v^{0}\right)}{\partial v}\right) \Delta v \text {, }
$$

here $u^{0} \leq u^{*} \leq u$ and $v^{0} \leq v^{*} \leq v=1-u$.

Assuming that the first derivatives within triangle area are finite one can then affirm there is a positive arbitrary constant $\theta$ which effects the following expressions

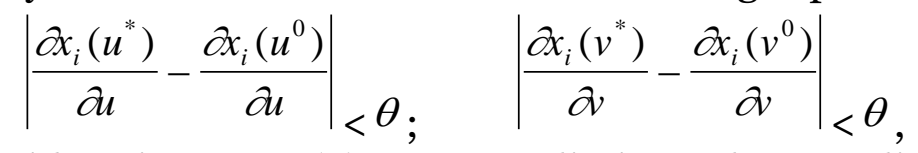

Taking into consideration eqn (9), we can limit $a$ the non-linear part to the following inequality

$$
\alpha_{i<} \theta(\Delta u+\Delta v)_{<} \theta \cdot P
$$

where $P$ is the plane triangle perimeter equal to $L_{1}+L_{2}+L_{3}$, i.e. the sum of its edges.

Comparing further two linear mappings (6), (7) one can indicate the difference between them which depends on the differences of the first derivatives in node 3 only. It means that if there is a positive constant $\beta$ then the following expression is true, i.e.

$$
\left|\hat{x}_{i}-x_{i}\right|_{<} \beta(\Delta u+\Delta v)_{<} \beta \cdot P,
$$

Taking into account (11) and (12), we can formulate the difference between non-linear mapping (8) and linear mapping (6) this way

where $\varphi \geq(\beta+\theta)$

$$
\left|\tilde{x}_{i}-x_{i}\right|_{<}(\beta+\theta) \cdot P<\varphi \cdot P
$$

Thus, the distance between images of similar points within curved triangle and plane triangle is not more than some constant, i.e.

$$
\sqrt{\sum_{i=1}^{3}\left(\tilde{x}_{i}-x_{i}\right)^{2}}<\sqrt{3} \varphi \cdot P
$$

A careful analysis of exp (14) enables to affirm that the difference between areas of curved triangle and plane triangle $\gamma$ is not more than the area of some tape similar area with width $\sqrt{3} \varphi \cdot P$ that is situated around of the perimeter of plane triangle. The area of this area is not more than

$$
\begin{array}{r}
\sqrt{3} \varphi \cdot P^{2}<\varepsilon \cdot \sum_{j=1}^{3} L_{j}^{2} \text { where } \varepsilon \geq \varphi \sqrt{3} \times P^{2} / \sum_{j=1}^{3} L_{j}^{2} \text {, or } \\
\gamma_{<} \varepsilon \cdot \sum_{j=1}^{3} L_{j}^{2} .
\end{array}
$$

It may be noted that $\varepsilon$ ratio depends only on the curvature of curved triangle and converges to zero as soon as curved triangle converges to the plane triangle. Taking into consideration the fact that the surface is approximated by the number of plane triangle elements, we may further write the following equation for the surface area

$$
\tilde{S}=\sum_{i=1}^{n}\left(\Delta_{i}+\gamma_{i}\right)
$$

where $\Delta_{i}$ - the area of a plane triangle number $I$,

$n-$ the total number of plane triangles.

$$
\gamma_{i}<\sum_{i=1}^{n} \varepsilon_{i} \sum_{p=1}^{k} L_{i p}^{2}
$$


here $\quad k$-a number of segments for the grid element $i$.

Assuming that all $\varepsilon_{i}$ factors are always $\varepsilon_{i} \leq \delta$ where $\delta>0$ we may write

$$
\widetilde{S}-\sum_{i=1}^{n} \Delta_{i}<\sum_{i=1}^{n} \varepsilon_{i} \sum_{p=1}^{k} L_{i p}^{2}<\delta \sum_{j=1}^{m} L_{j}^{2}
$$

where $\delta$-ratio depends on the curvature of only the largest grid segment and converges to zero as soon as it becomes smaller, i.e.

$$
\widetilde{S}-\lim _{n \rightarrow \infty} \sum_{i=1}^{n} \Delta_{i}=0 \text {, when } \lim _{n \rightarrow \infty} \delta=0 \text {, }
$$

In other words, refining the mesh so that to condense the grid cells one can converge the area of the poly-plane cellular structure to the exact surface area as close as possible. The next step is to prove that the functional grid area can be replaced by the functional sum of the whole grid segment length. Let's further introduce the function $\tilde{L}=\sum_{j=1}^{3} \tilde{L}_{j}^{2}$ where $\tilde{L}_{j}^{2}$ the length of an edge number $j$ of a curved cell (see Fig.3) which is defined by the following expression (see works [9], [10])

$$
\tilde{L}_{j}^{2}=\left(\int_{t_{0}}^{t} \sqrt{E \cdot u^{\prime 2}+2 F \cdot u^{\prime} \cdot v^{\prime}+G \cdot v^{\prime 2}} d t\right)^{2}
$$

where $E, F, G$ - metric ratios of the first surface quadratic form,

$t-$ the $1 \mathrm{D}$ edge parameter of the triangle.

One can notice the following obvious inequality

$$
\sum_{j=1}^{m} L_{j}^{2} \sum_{j=1}^{m} \tilde{L}_{j}^{2}
$$

where $m$ - the total number of grid edges.

Taking into account the analysis of the mesh area convergence to the exact surface area, we can estimate the difference between the curved grid segment number $j$ and its linear approximation as the following inequality similar to exp (15)

$$
\widetilde{L}_{j}^{2}-L_{j}^{2}<4 \varepsilon_{j} \cdot\left(\varepsilon_{j}+L_{j}\right),
$$

Exp. (22) demonstrates that the function $\sum_{j=1}^{m} L_{j}^{2}$ converges to the function $\sum_{j=1}^{m} \tilde{L}_{j}^{2}$ due to the mesh refinement. This happens in parallel to the vanishing of the function (18). Now we can see how exp. (22) transforms into the following equality due to $n \rightarrow \infty$ and $m \rightarrow \infty$, i.e.

$$
\lim _{m \rightarrow \infty} \sum_{j=1}^{m} L_{j}^{2} \quad \lim _{m \rightarrow \infty} \sum_{j=1}^{m} \tilde{L}_{j}^{2}
$$

Thus, due to (2) and (23) we can apply (1) to minimal surface form finding as accurate as the triangle grid is finely condensed. Besides, the expression (18) is very similar to (2) and it is the necessary condition for functional (1) applicability.

\section{Basic Minimum Surface Feature}

\section{The preliminary notation}

In previous chapter we have showed that there are two functions $\Delta$ - the approximate surface area and $\sum_{j=1}^{m} L_{j}^{2}$ - some function associated with a sum of the linear length of surface mesh segments. Both functions converge to stable values $\tilde{S}$ and $\tilde{L}^{2}$ simultaneously due to the mesh refinement to condense a grid cell. Obviously, function $\widetilde{L}^{2}$ depends on only the surface of the first quadratic form. If $3 \mathrm{D}$ surface is bounded by a closed fixed contour and can be 
modified to converge to a surface with a minimum area, then such statement about $\tilde{L}^{2}$ feature is true as well. In this case, we can formulate the problem like this: what is the rule for the behavior of the first quadratic form surface due to the surface variation? It seems to be a basic feature of the minimum surfaces. If such feature is true, it serves as a clear proof of function (1) as a sufficient condition for minimal surface form finding. Now we can formulate the theorem.

\section{Theorem:}

If an arbitrary regular surface bounded by an arbitrary $3 D$ closed fixed contour converges to the minimum area, then the first surface quadratic form converges at any surface point to the minimum as well.

\section{Proof}

Let us consider that an arbitrary regular surface is approximated by an arbitrary curved grid with infinite small cells and the surface together with all surface derivations are continuously independent on the surface deformation. In such a case we can insert a local surface FEMsimilar $u, v$ parameterization as described in the previous chapter (see Fig. 3) and define the surface co-ordinates within any cell with the following functions determine surface coordinates in any cell using the following functions

$$
x_{i}=X_{i}\left(u, v, \Delta x_{i}\right), \quad i=1,2,3
$$

where $\Delta x_{i}$ - some increment vector of nodal co-ordinates and can be various to get the minimum surface area,

$I$ - the number of co-ordinate axis.

Let us assume further that there is some $\Delta x_{o}$-increment vector of grid nodal co-ordinates, which provides the minimum for function $\tilde{S}(\Delta x)$. To find it, we have to give derivation of $\widetilde{S}(\Delta x)$ by vector $\Delta x$ in the following way

$$
\frac{\partial \tilde{S}\left(X_{i}\right)}{\partial\left(\Delta x_{i}\right)}=0
$$

It is well- known that the surface area may be written as the following form (see [9], [10])

$$
\widetilde{S}=\sum_{i=1}^{n} \int_{\Delta_{i}} \sqrt{E G-F^{2}} d u \cdot d v
$$

One should indicate that the minimum condition (25) taking into account eqn. (26) leads the following local minimum condition for the area of infinitely small element at an arbitrary surface point

$$
E \frac{\partial G(X)}{\partial(\Delta x)}+G \frac{\partial E(X)}{\partial(\Delta x)}-2 F \frac{\partial F(X)}{\partial(\Delta x)}=0
$$

Considering that $E, F, G$ are metric ratios that are expressed with the forms (see [9], [10])

$$
\begin{aligned}
& E=\left(\frac{\partial X_{1}}{\partial u}\right)^{2}+\left(\frac{\partial X_{2}}{\partial u}\right)^{2}+\left(\frac{\partial X_{3}}{\partial u}\right)^{2} \\
& G=\left(\frac{\partial X_{1}}{\partial v}\right)^{2}+\left(\frac{\partial X_{2}}{\partial v}\right)^{2}+\left(\frac{\partial X_{3}}{\partial v}\right)^{2} \\
& F=\frac{\partial X_{1}}{\partial u} \cdot \frac{\partial X_{1}}{\partial v}+\frac{\partial X_{2}}{\partial u} \cdot \frac{\partial X_{2}}{\partial v}+\frac{\partial X_{3}}{\partial u} \cdot \frac{\partial X_{3}}{\partial v}
\end{aligned}
$$

we may write further three simultaneous minimum conditions on the basis of exp. (25) 


$$
\begin{aligned}
& {\left[X_{2}^{u} \frac{\partial X_{1}^{v}}{\partial\left(\Delta x_{1}\right)}-X_{2}^{v} \frac{\partial X_{1}^{u}}{\partial\left(\Delta x_{1}\right)}\right] \cdot\left(X_{1}^{v} \cdot X_{2}^{u}-X_{1}^{u} \cdot X_{2}^{v}\right)+} \\
& +\left[X_{3}^{u} \frac{\partial X_{1}^{v}}{\partial\left(\Delta x_{1}\right)}-X_{3}^{v} \frac{\partial X_{1}^{u}}{\partial\left(\Delta x_{1}\right)}\right] \cdot\left(X_{1}^{v} \cdot X_{3}^{u}-X_{1}^{u} \cdot X_{3}^{v}\right)=0 \\
& {\left[X_{1}^{v} \frac{\partial X_{2}^{u}}{\partial\left(\Delta x_{2}\right)}-X_{1}^{u} \frac{\partial X_{2}^{v}}{\partial\left(\Delta x_{2}\right)}\right] \cdot\left(X_{1}^{v} \cdot X_{2}^{u}-X_{1}^{u} \cdot X_{2}^{v}\right)+} \\
& +\left[X_{3}^{u} \frac{\partial X_{2}^{v}}{\partial\left(\Delta x_{2}\right)}-X_{3}^{v} \frac{\partial X_{2}^{u}}{\partial\left(\Delta x_{2}\right)}\right] \cdot\left(X_{2}^{v} \cdot X_{3}^{u}-X_{2}^{u} \cdot X_{3}^{v}\right)=0 \\
& {\left[X_{1}^{u} \frac{\partial X_{3}^{v}}{\partial\left(\Delta x_{3}\right)}-X_{3}^{v} \frac{\partial X_{3}^{u}}{\partial\left(\Delta x_{3}\right)}\right] \cdot\left(X_{1}^{u} \cdot X_{3}^{v}-X_{1}^{v} \cdot X_{3}^{u}\right)+} \\
& +\left[X_{2}^{u} \frac{\partial X_{3}^{v}}{\partial\left(\Delta x_{3}\right)}-X_{2}^{v} \frac{\partial X_{3}^{u}}{\partial\left(\Delta x_{3}\right)}\right] \cdot\left(X_{2}^{u} \cdot X_{3}^{v}-X_{2}^{v} \cdot X_{3}^{u}\right)=0
\end{aligned}
$$

One can note that the determinants within round brackets of conditions (29) are not close to zero at an arbitrary regular surface point simultaneously, i.e., an equation system

$$
\left(\begin{array}{ccc}
a_{11} & a_{12} & 0 \\
a_{21} & 0 & a_{23} \\
0 & a_{32} & a_{33}
\end{array}\right) \cdot\left\{\begin{array}{l}
b_{1} \\
b_{2} \\
b_{3}
\end{array}\right\}=\left\{\begin{array}{l}
0 \\
0 \\
0
\end{array}\right\}
$$

where $a_{i j}$ - the expressions in quadrangular brackets of (29), vector $b$,

$b_{i}$ - the expressions in round brackets of (29), always receives a non-zero solution for

always gets a non-zero solution with respect to vector $b$, only if $\operatorname{det}\left[a_{i j}\right]=0$. Assuming that $\operatorname{det}\left[a_{i j}\right]=0$ is always true, we can write

$$
-a_{32} a_{23} a_{11}-a_{33} a_{21} a_{12}=0
$$

or

$$
\begin{aligned}
& {\left[X_{2}^{v} \frac{\partial X_{1}^{u}}{\partial\left(\Delta x_{1}\right)}-X_{2}^{u} \frac{\partial X_{1}^{v}}{\partial\left(\Delta x_{1}\right)}\right] \cdot\left[X_{3}^{v} \frac{\partial X_{2}^{u}}{\partial\left(\Delta x_{2}\right)}-X_{3}^{u} \frac{\partial X_{2}^{v}}{\partial\left(\Delta x_{2}\right)}\right] \cdot\left[X_{1}^{v} \frac{\partial X_{3}^{u}}{\partial\left(\Delta x_{3}\right)}-X_{1}^{u} \frac{\partial X_{3}^{v}}{\partial\left(\Delta x_{3}\right)}\right]-} \\
& -\left[X_{3}^{v} \frac{\partial X_{1}^{u}}{\partial\left(\Delta x_{1}\right)}-X_{3}^{u} \frac{\partial X_{1}^{v}}{\partial\left(\Delta x_{1}\right)}\right] \cdot\left[X_{1}^{v} \frac{\partial X_{2}^{u}}{\partial\left(\Delta x_{2}\right)}-X_{1}^{u} \frac{\partial X_{2}^{v}}{\partial\left(\Delta x_{2}\right)}\right] \cdot\left[X_{2}^{v} \frac{\partial X_{3}^{u}}{\partial\left(\Delta x_{3}\right)}-X_{2}^{u} \frac{\partial X_{3}^{v}}{\partial\left(\Delta x_{3}\right)}\right]=0
\end{aligned}
$$

One can indicate that (31) leads to the following most general condition, independent of the location of a surface point

$$
\begin{aligned}
& \frac{\partial X_{1}^{u}}{\partial\left(\Delta x_{1}\right)}=\frac{\partial X_{1}^{v}}{\partial\left(\Delta x_{1}\right)} \\
& \frac{\partial X_{2}^{u}}{\partial\left(\Delta x_{2}\right)}=\frac{\partial X_{2}^{v}}{\partial\left(\Delta x_{2}\right)} \\
& \frac{\partial X_{3}^{u}}{\partial\left(\Delta x_{3}\right)}=\frac{\partial X_{3}^{v}}{\partial\left(\Delta x_{3}\right)} .
\end{aligned}
$$

If conditions (32) are always true at any/every surface point then the system of equations (29) always gets a non-zero solution and conditions (32) are sufficient and necessary for the system (29) to be correct.

Let us transform the further system of equations (29) taking into account conditions (32). After all transformations we can figure out the following equation system equivalent to (29) 


$$
\begin{aligned}
& \frac{\partial X_{1}^{v}}{\partial\left(\Delta x_{1}\right)} \cdot\left[X_{1}^{v} \cdot(E-F)+X_{1}^{u} \cdot(G-F)\right]=0 \\
& \frac{\partial X_{2}^{v}}{\partial\left(\Delta x_{2}\right)} \cdot\left[X_{2}^{v} \cdot(E-F)+X_{2}^{u} \cdot(G-F)\right]=0 \\
& \frac{\partial X_{3}^{v}}{\partial\left(\Delta x_{3}\right)} \cdot\left[X_{3}^{v} \cdot(E-F)+X_{3}^{u} \cdot(G-F)\right]=0
\end{aligned}
$$

where $E, F, G$-the metric ratios of the surface of the first quadratic form as earlier.

The functions in quadrangular brackets of (33) have no non-zero values simultaneously because a parameter of basis $u, v$ is non-singular. Therefore, both equation systems (33) and (29) solution can be defined as the following equation system similar to (32)

$$
\begin{aligned}
& \frac{\partial X_{1}^{u}}{\partial\left(\Delta x_{1}\right)}=\frac{\partial X_{1}^{v}}{\partial\left(\Delta x_{1}\right)}=0 ; \\
& \frac{\partial X_{2}^{u}}{\partial\left(\Delta x_{2}\right)}=\frac{\partial X_{2}^{v}}{\partial\left(\Delta x_{2}\right)}=0 ; \\
& \frac{\partial X_{3}^{u}}{\partial\left(\Delta x_{3}\right)}=\frac{\partial X_{3}^{v}}{\partial\left(\Delta x_{3}\right)}=0 .
\end{aligned}
$$

This means that as soon as function $\tilde{S}(\Delta x)$ converges to minimum the first co-ordinate derivatives converge to minimum simultaneously at any surface point.

The first surface quadratic form can be expressed by the following equation for an arbitrary curve arc differential at an arbitrary surface point [8], [9] The first surface quadratic form can be expressed by the following equation for an arbitrary curve arc differential at an arbitrary surface point [8], [9]

$$
(d s)^{2}=E \cdot d u^{2}+2 F \cdot d u \cdot d v+G \cdot d v^{2} .
$$

The local minimum conditions similar to (25) for function (35) can be written as the following three simultaneous equations

$$
\begin{aligned}
& {\left[\frac{\partial X_{1}^{u}}{\partial\left(\Delta x_{1}\right)} d u+\frac{\partial X_{1}^{v}}{\partial\left(\Delta x_{1}\right)} d v\right] \cdot d X_{1}=0} \\
& {\left[\frac{\partial X_{2}^{u}}{\partial\left(\Delta x_{2}\right)} d u+\frac{\partial X_{2}^{v}}{\partial\left(\Delta x_{2}\right)} d v\right] \cdot d X_{2}=0} \\
& {\left[\frac{\partial X_{3}^{u}}{\partial\left(\Delta x_{3}\right)} d u+\frac{\partial X_{3}^{v}}{\partial\left(\Delta x_{3}\right)} d v\right] \cdot d X_{3}=0}
\end{aligned}
$$

or in the vector form

$$
\begin{aligned}
& \left(\frac{\partial(d \overrightarrow{\mathbf{r}})}{\partial\left(\Delta x_{1}\right)}, d \overrightarrow{\mathbf{r}}\right)=0 ; \\
& \left(\frac{\partial(d \overrightarrow{\mathbf{r}})}{\partial\left(\Delta x_{2}\right)}, d \overrightarrow{\mathbf{r}}\right)=0 ; \\
& \left(\frac{\partial(d \overrightarrow{\mathbf{r}})}{\partial\left(\Delta x_{3}\right)}, d \overrightarrow{\mathbf{r}}\right)=0 .
\end{aligned}
$$

It should be noted that three scalar products of the same vector $d \overrightarrow{\mathbf{r}} \neq 0$ with three different vectors $\frac{\partial(d \overrightarrow{\mathbf{r}})}{\partial\left(\Delta x_{1}\right)}, \frac{\partial(d \overrightarrow{\mathbf{r}})}{\partial\left(\Delta x_{2}\right)}, \frac{\partial(d \overrightarrow{\mathbf{r}})}{\partial\left(\Delta x_{3}\right)}$ are equal to zero simultaneously if either the derivation vectors are situated at the same plane normally to vector $d \overrightarrow{\mathbf{r}} \neq 0$. 
However, the latter is impossible because the vector product does not always equal to zero if the derivation of the vectors are non-zero vectors or they are equal to zero simultaneously. On the other hand, three different derivation of the vectors $\frac{\partial(d \overrightarrow{\mathbf{r}})}{\partial\left(\Delta x_{1}\right)}, \frac{\partial(d \overrightarrow{\mathbf{r}})}{\partial\left(\Delta x_{2}\right)}, \frac{\partial(d \overrightarrow{\mathbf{r}})}{\partial\left(\Delta x_{3}\right)}$ are always equal to zero only if system (34) is true. In fact, their components

$$
\begin{aligned}
& \frac{\partial X_{1}^{u}}{\partial\left(\Delta x_{1}\right)} d u+\frac{\partial X_{1}^{v}}{\partial\left(\Delta x_{1}\right)} d v=0 ; \\
& \frac{\partial X_{2}^{u}}{\partial\left(\Delta x_{2}\right)} d u+\frac{\partial X_{2}^{v}}{\partial\left(\Delta x_{2}\right)} d v=0 ; \\
& \frac{\partial X_{3}^{u}}{\partial\left(\Delta x_{3}\right)} d u+\frac{\partial X_{3}^{v}}{\partial\left(\Delta x_{3}\right)} d v=0 .
\end{aligned}
$$

are total differentials and always equal to zero if all local derivations are equal simultaneously to zero too due to arbitrary values of $d u$ and $d v$. Therefore, we can obtain the same equation system (34).

It means that the minimum condition of the first quadratic form $(d s)^{2}$ is also equivalent to equation system (34) and both functions $\tilde{S}(\Delta x)$ and $(d s)^{2}$ converge to their minima on the same incremental vector. Besides, one can also be convinced that vector $\Delta x_{o}$ will obviously provide the minimum for $E, F, G$ - metric ratios simultaneously at the same $\Delta x_{o}$-vector, i.e.

$$
\frac{\partial E\left(X_{i}\right)}{\partial\left(\Delta x_{i}\right)}=\frac{\partial G\left(X_{i}\right)}{\partial\left(\Delta x_{i}\right)}=\frac{\partial F\left(X_{i}\right)}{\partial\left(\Delta x_{i}\right)}=0
$$

Thus, if the surface area near an arbitrary surface point converges to minimum then the first quadratic form near this point converges to minimum too because their minimum conditions are equivalent to equation system (34) for their finding minima. One can make the same conclusion at every regular surface point. So, we have proved the theorem. Actually, expression (39) is a sufficient condition for functional (1) applicability.

\section{Applications}

Now we are convinced that SGM allows minimizing the surface embedded into non-planed and planed, closed contours. From the physical point of view, this condition reflects the total energy balance of the nodal system.

When designing the surface of the tent structure, it is vital to have it suspended on the special Frame object. Frame object is a set of spatial edges connected with each other. One can model edges by segments of straight lines or arcs of a circle. The Frame object is the basis for the tent cloth. The Frame edges and nodes should correspond to the tent cloth constrains. The system accepts topological triangles or as cloth preliminary patches [11].The algorithm for finding form of minimal and similar surfaces is implemented in the $\mathrm{K}_{3}$-Tent tensile fabric $\mathrm{CAD}$ system [12].The $\mathrm{K}_{3}$-Tent system allows a designer to choose the form-finding of tent structure of any complexity, supports the technology of its manufacture, including cutting pattern generation, setting of allowances for the pattern etc. The $\mathrm{K}_{3}$-Tent system is developed in $\mathrm{C}++$ language environment under Windows $10 \mathrm{OS}$ and is a commercial product for small and medium-sized businesses.

The K3-Tent system allows the designer to choose the shape of the tent structure of any complexity, supports the technology of its manufacture, including the formation of a pattern, setting of allowances for the pattern, etc.

The Frame concept is widely used in the process for form finding tent structures. In Fig.4 one can see the surface of catenoid as an example of the developed approach.The two rings take on the role of the Frame to model the surface. The radii of the rings and the height of the 
catenoid are equal to 1.o. The numerical area of the catenoidal surface is equal to 2,99671890145 (exact value is 2.992).

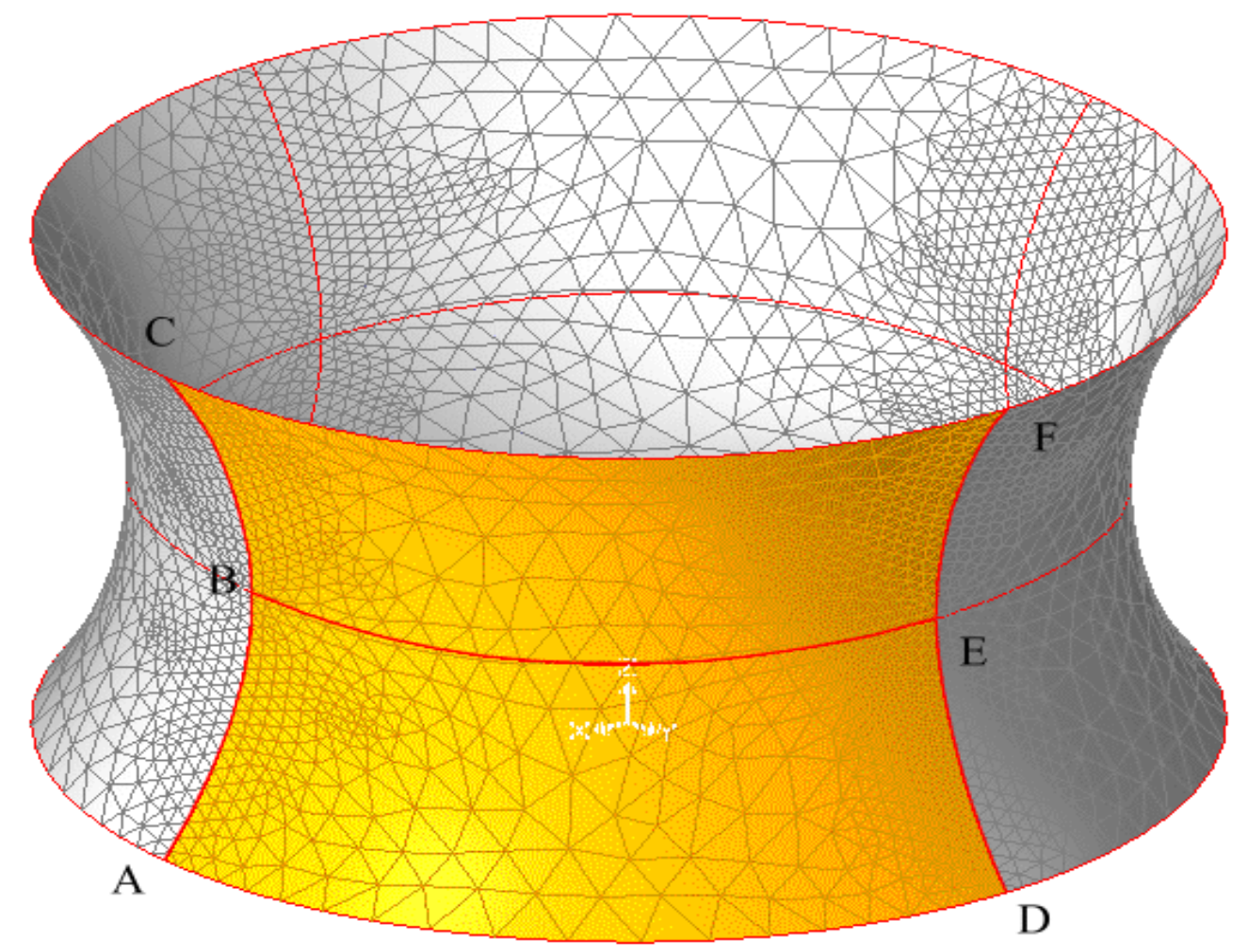

Figure 4. Catenoidal surface

The second problem is that of a helix surface (Fig. 5) between two concentric cylinders with a radii of 0.5 and 1.0 respectively and surface step $H=1.0$. The numerical area is 2.41043 (exact value 2.41062).

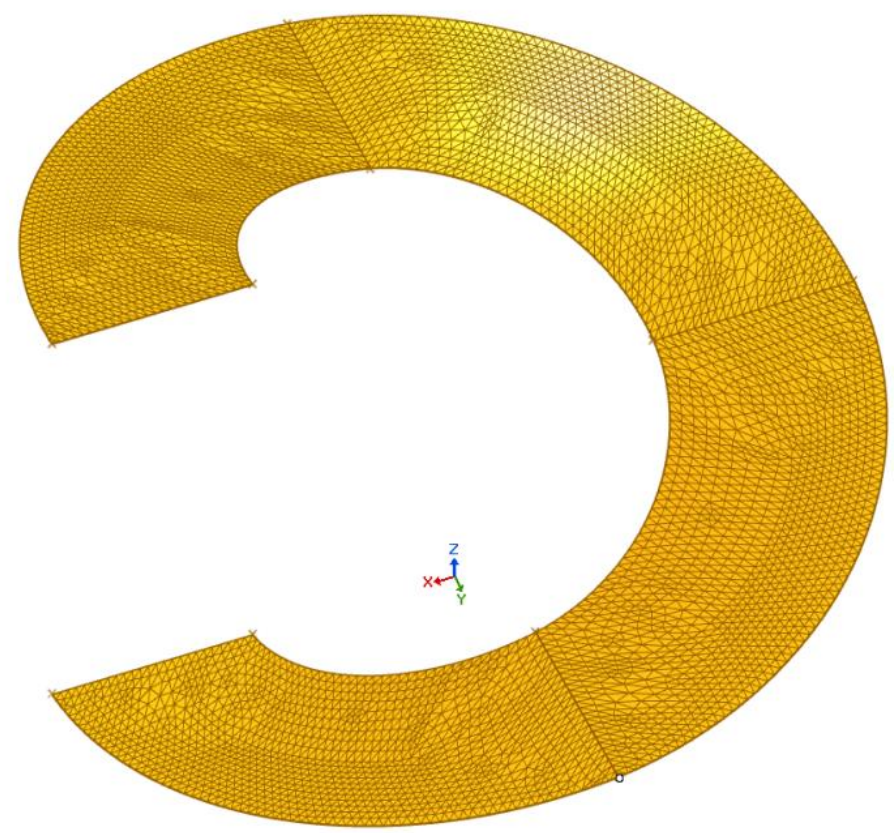

Figure 5. Helix surface 
The following examples illustrate the form of the Hypar (Hyperbolic Parabolic) tent (see Fig. 6) and tent roof of Entertainment Center (Fig. 7).

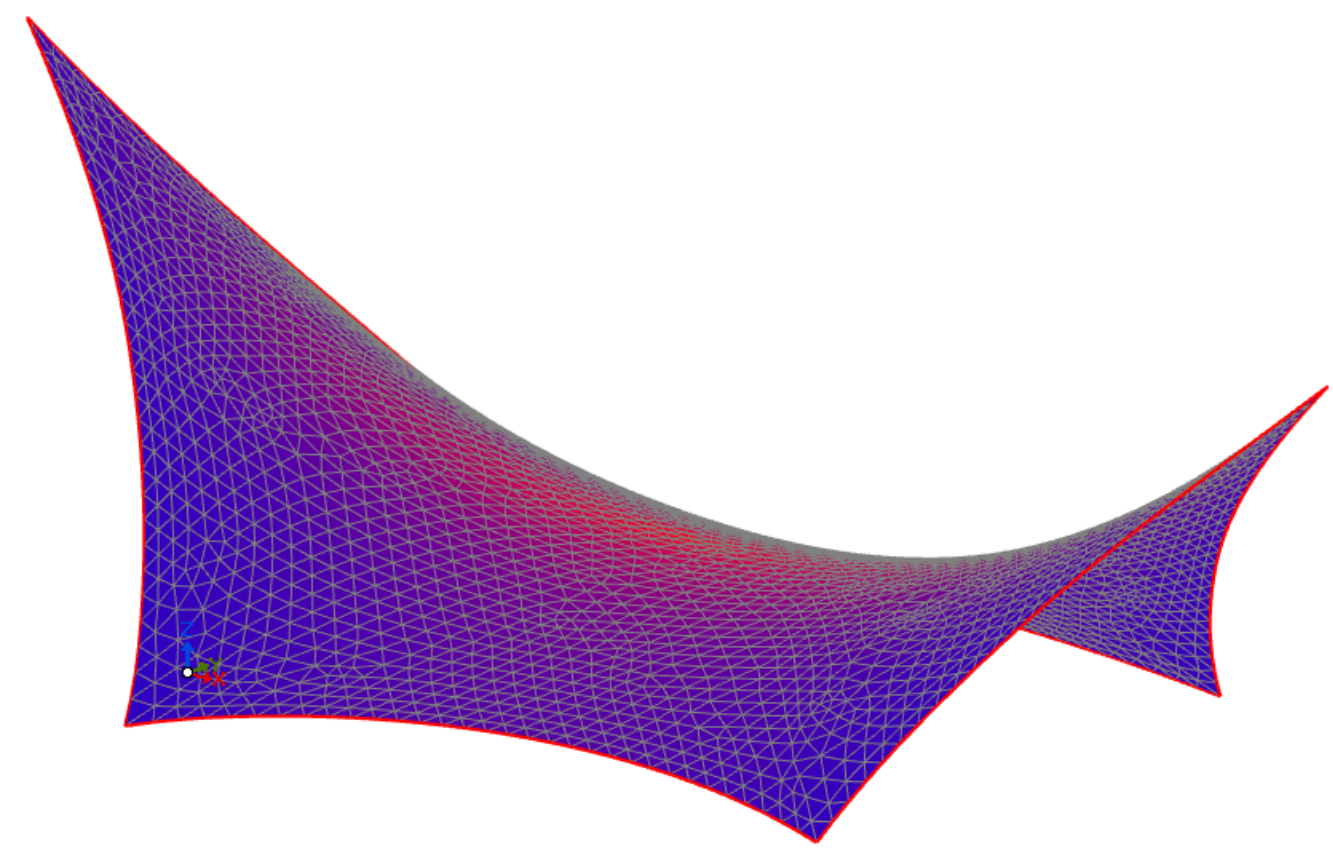

Figure 6. Hyperbolic parabolic tent (Hypar)

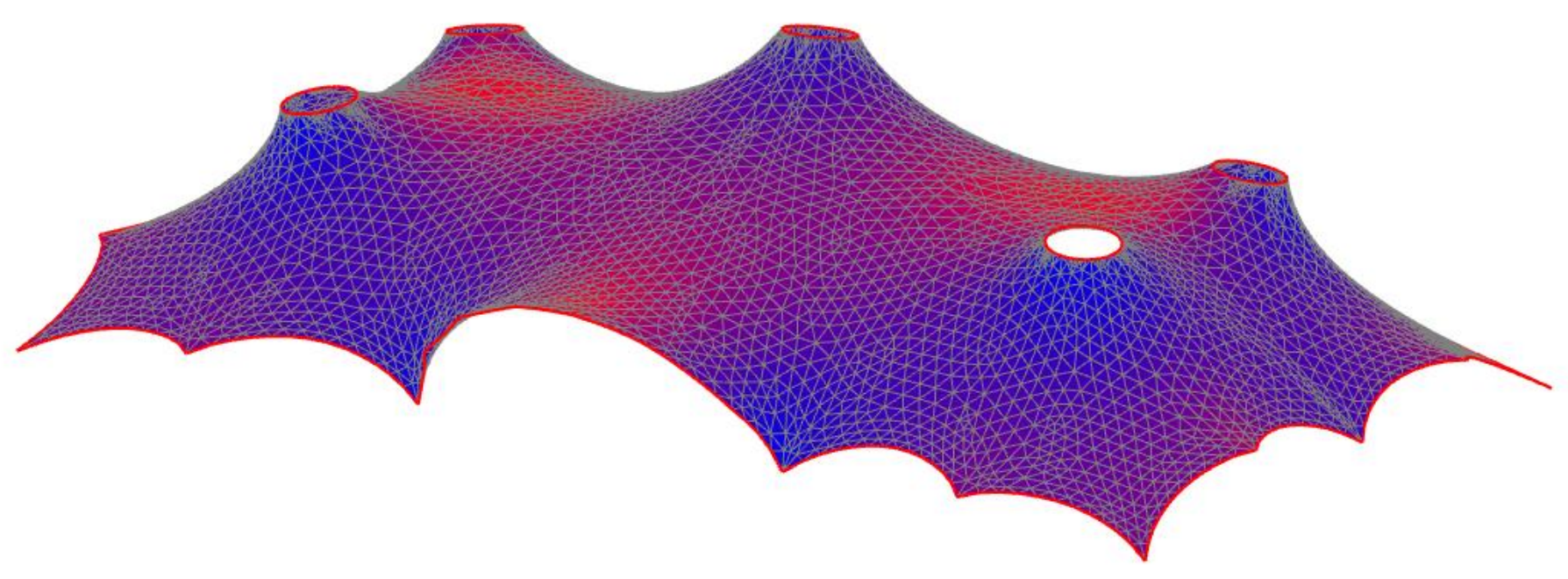

Figure 7. Roof of Entertainment Center

\section{Conclusion}

Now we can make some conclusions, namely:

First, both functions $\widetilde{S}$ and $\tilde{L}$ get the same minimum due to the surface variation because $\tilde{L}$ -function is the sum of the integrals of the surface first quadratic form along the curved segments of mesh (as shown in Section 3). Thus, the minimum condition $\frac{\partial \tilde{S}}{\partial(\Delta x)}=0$ we can 
replace by $\frac{\partial \tilde{L}}{\partial(\Delta x)}=0$ condition, because it gives the same $\Delta x_{o}$-vector. As it is shown in [6], this permits obtaining a much easier solution.

Second, if the surface mesh is fine enough to neglect the curvature of grid cells, then both conditions $\frac{\partial \tilde{S}}{\partial(\Delta x)}=0$ and $\frac{\partial \tilde{L}}{\partial(\Delta x)}=0$ can be substituted by conditions $\frac{\partial \Delta}{\partial(\Delta x)}=0$ and $\frac{\partial L}{\partial(\Delta x)}=0$. Here $L$-function is identical to $\Pi$-function (see eqn.(1)and [7]), respectively, due to eqns. (19), (23). If the mesh is not fine and differences $\tilde{S}-\sum_{i=1}^{n} \Delta_{i}$ and $\widetilde{L}_{j}^{2}-\bar{L}_{j}^{2}$ (see exp. (22)) cannot be neglected, all three functions (say $\tilde{S}, \Delta$ и $L$ - functions) have minima with different co-ordinate increments, and the technique described in [6] can be applied to minimum surface form finding, approximately due to very crude mesh. One can find the illustration of this case as a sample for the rectangular area in Fig.2.

Third, the described technique is independent on the type of mesh, which can be as uniform as transient.

And finally, the implemented algorithm based on the Stretched Grid Method automatically provides the optimal surface form, close to minimal area, without any folders, beads or plaiting etc.

\section{Acknowledgement}

The work was supported by the Russian Foundation for Basic Research, RFBR grant № 1907-01024.

\section{References}

[1] William H. Meeks III, Joaquín Pérez, A survey on classical minimal surface theory. Bull. Amer. Math. Soc. 48 (2011), 325-407.DOI: 10.1090/So273-0979-2011-01334-9 http://www.ugr.es/ jperez/papers/monograph-book2.pdf

[2] E.G. Grigorieva, A.A. Klyachin, V.A. Klyachin, Boundary identification algorithms and visualization of the solution of the Plateau problem in a Blender environment, Scientific Visualization 9(4) 2017, p.13-25, http://sv-journal.org/2017-4/o2.php?lang=en.

[3] B.Tabarrok, Z.Qin. Form Finding and Cutting Pattern Generation for Fabric Tension Structures, -Microcomputers in Civil Engineering J., № 8, 1993, pp.377-384.

[4] Tabarrok, Y.Xiong. Some Variational Formulations for minimum surface. Acta Mechanica, vol.89/1-4, 1991, pp.33-43.

[5] Tabarrok B., Qin Z. Nonlinear analysis of tension structures. // Computers and Structures, N 45, 1992 pp.973-984.

[6] Tabarrok B., Qin Z. A finite element procedure for form finding of tension structures. // The Transactions of the Canadian Society for Mechanical Engineering, N 16, 1992 pp.235250.

[7] E.V.Popov, On Some Variational Formulations for Minimum Surface- Transactions of Canadian Society of Mechanics for Engineering, Univ. of Alberta, vol.20, N 4, 1997

[8] Qian Jian-hua, Application of a Variable-Resolution Stretched Grid to a Regional Atmospheric Model with Physics Parameterization, Advances in Earth Science 2007, 22(11) $1185^{-1190}$

[9] C.A.Korn and T.M.Korn. Mathematical Handbook for scientists and engineers. Definitions, Theorems and Formulas for reference and review. McGraw-Hill, Book Company Inc., New York Toronto London, 1961 
[10] Hazewinkel, Michiel, ed., Differential geometry, Encyclopedia of Mathematics, Springer, (2001) ISBN 978-1-55608-010-4

http://www.encyclopediaofmath.org/index.php/Differential_geometry

[11] E.V. Popov, et al, Tensile Structure Form-Finding on the Basis of Properties of FrameGrid Template // ICGG 2018 - Proceedings of the 18th International Conference on Geometry and Graphics. ICGG 2018. Advances in Intelligent Systems and Computing, pp. 1391-1403

[12] E.V. Popov, et al, Software package for membrane tent structures designing "K3-Tent" version 8.o. Certificate of a computer program RU 2020619455, 17/08/2020. Application No. 2020618655 of $06 / 08 / 2020$. 